\title{
Bioamplificador de Alta Resolución para Electroencefalografía de Amplio Espectro
}

\section{High-resolution bioamplifier for broad-spectrum electroencephalography}

\begin{abstract}
Nelson Dugarte Jerez.
Instituto Regional de Bioingeniería (IRB) - Centro Regional de Investigación y desarrollo en Computación y Neurociencia (CeReCoN), Facultad Regional Mendoza (FRM), Universidad Tecnológica Nacional (UTN) - Argentina

ndj0227@gmail.com
\end{abstract}

\section{Antonio Alvarez Abril.}

Instituto Regional de Bioingeniería (IRB) - Centro Regional de Investigación y desarrollo en Computación y Neurociencia (CeReCoN), Facultad Regional Mendoza (FRM), Universidad Tecnológica Nacional (UTN) - Argentina antonioalvarezabril@yahoo.com.ar

\section{Edison del C. Dugarte D.}

Grupo de Ingeniería Biomédica (GIBULA), Universidad de Los Andes (ULA), Mérida, Venezuela. edinson0909@gmail.com

\section{Negman W. Alvarado Riviera.}

Instituto Regional de Bioingeniería (IRB) - Centro Regional de Investigación y desarrollo en Computación y Neurociencia (CeReCoN), Universidad Tecnológica Nacional (UTN), Facultad Regional Mendoza (FRM).

neuroservices.tt@gmail.com

\section{Resumen}

En este artículo se presenta el desarrollo de la etapa de hardware de un bioamplificador multicanal, de alta resolución y bajo ruido, diseñado para adquirir 8 canales de las señales de biopotencial emitidas por el cerebro humano. Básicamente es un electroencefalógrafo (EEG) de 8 derivaciones, pero diseñado para captar las señales con ancho de banda ampliado, en comparación con los EEG comunes. Este bioamplificador se realiza con el propósito de adquirir señales de sectores específicos del cerebro, para estudiar patrones de frecuencia superiores a los rangos comúnmente analizados en la electroencefalografía convencional. 
El producto obtenido está formado por 8 circuitos idénticos acoplados para captar todas las señales con respecto a un electrodo central. Cada circuito consiste en un amplificador diferencial con circuitos de realimentación del voltaje en modo común, que permiten mejorar la relación de rechazo al ruido. Se utilizan circuitos de acoplamiento AC de alta eficiencia para reducción del corrimiento de línea de base sin alterar la señal. Cada derivación tiene ganancia de 10000, para obtener niveles de amplitud en el orden de Volts a partir de las señales captadas en el orden de los micro-Volts. Los resultados preliminares muestran que el sistema funciona eficientemente dentro de un rango de 0.5 a $300 \mathrm{~Hz}$, con niveles de error en la medición de amplitud inferiores al 0.14\%.

Palabras Clave: Amplificador de biopotencial - Señal electroencefalográfica - EEG con ancho de banda ampliado - Desarrollo de hardware.

\begin{abstract}
This article presents the hardware development stage of a high-resolution and low-noise multi-channel bioamplifier, designed to acquire 8 channels of biopotential signals by human brain emitted. Basically it's an 8-lead electroencephalograph (EEG), but designed to pick up signals with wide bandwidth, compared to common EEGs. This bioamplifier is made with the purpose of acquire signals from specific brain sectors, for frequency patterns study higher than the ranges commonly analyzed in conventional electroencephalography. The obtained product consists of 8 identical circuits coupled to capture all the signals with respect to a central electrode. Each circuits consists of a differential amplifier with common mode voltage feedback circuits, which allow improve the noise rejection ratio. High efficiency AC coupling circuits are used to reduce baseline shift without signal altering. Each lead has gain of 10000, to obtain amplitude levels in the Volts order from the signals captured in micro-Volts order. Preliminary results show that the system operates efficiently within a range of 0.5 to $300 \mathrm{~Hz}$, with amplitude measurement error levels less than $0.1 \%$.
\end{abstract}

Keywords: Biopotential amplifier - Electroencephalographic signal - EEG with extended bandwidth - Hardware development.

\title{
1 Introducción
}

La evolución del sistema nervioso ha representado uno de los pilares fundamentales en el desarrollo de los seres vivos. Desde la interconectividad entre las células hasta el desarrollo de las emociones, depende del funcionamiento de las redes neuronales. Y del trabajo en conjunto entre estas redes se destaca el funcionamiento del cerebro (Ganong, 1999).

El estudio de las funciones cognitivas y de redes nerviosas, así como las investigaciones que se realizan para detectar las patologías que lo afectan, se realizan con la implementación de instrumentación que permita ver el comportamiento de las estructuras neuronales (Tucci, 2004), (Organización Mundial de la Salud, 2006).

Las células presentan la característica de producir potenciales bioeléctricos, como resultado de la actividad electroquímica de sus membranas (Ganong, 1999). Ejemplos de este tipo de células son las neuronas (Tucci, 2004). Las señales que se generan en las neuronas corresponden a un tipo particular de biopotencial, que presenta cambios importantes cuando se activan (despolarización y repolarización) (Ganong, 1999). El resultado de la sumatoria de 
los biopotenciales generados en las redes neuronales se desplazan por todo el organismo, y se pueden captar y medir en el exterior del cuerpo en forma de vectores de potencial en función del tiempo. Cada vector se denomina en la jerga médica como derivación (Navarro, 2008).

Para captar las señales de biopotencial originadas en el cerebro se colocan los electrodos sobre el cuero cabelludo según el sistema internacional o sistema 10-20, llamado así porque los electrodos se encuentran espaciados entre el 10\% y el 20\% de la distancia entre los puntos que limitan el contenido del cerebro sobre la superficie del cráneo (Navarro, 2008), (Morillo, 2005). El instrumento que se utiliza para captar estas señales se denomina electroencefalógrafo y el estudio de las derivaciones que se obtienen se denomina electroencefalograma o EEG (Morillo, 2005).

Los potenciales que se captan con los electroencefalógrafos comunes están en el orden de los microvoltios $(\mu \mathrm{V})$ y se encuentran en el rango de frecuencias de entre 0.5 y $70 \mathrm{~Hz}$ (Navarro, 2008) (Morillo, 2005). Ahora resulta interesante realizar la adquisición de señales EEG en investigaciones sobre la biodinámica emocional, analizando la respuesta de frecuencia hasta $300 \mathrm{~Hz}$. El problema que se presenta cuando se desea captar este tipo de señales, se debe a los requerimientos de amplificación de señales en el orden de los $\mu \mathrm{V}$ con alto grado de fiabilidad en presencia de niveles de ruido que pueden superar los 200 mili Volts $(\mathrm{mV})$ (Tucci, 2004) (Morris, 2001). Esto trae como consecuencia que los instrumentos para EEG con acho de banda amplio, del tipo comercial, sean relativamente costosos.

También es evidente que las empresas que desarrollan este tipo de hardware protegen sus equipos produciendo aparatos con tecnología cerrada, donde es casi imposible realizar cambios que modifiquen el hardware de los instrumentos para ser implementados en aplicaciones que no sean específicamente las propuestas por el fabricante del producto.

Estudios realizados desde el año 2015 en el IRB y en GIBULA, han generado una serie de productos de innovación tecnológica en cuanto al hardware de adquisición de biopotenciales de alta resolución (Dugarte et al., 2012), (Alvarez et al., 2019), que sustentan la base técnica para el desarrollo de este proyecto por medio de la experiencia adquirida. Los antecedentes en nuestros centros de investigación también han demostrado que se puede obtener un índice que relaciona la señal MU con la respuesta emocional (Alvarez et al., 2019), (Dugarte et al., 2018).

\section{Materiales y métodos}

El sistema que se requiere para realizar la adquisición de la señal EEG consta de tres partes: el bioamplificador, la tarjeta de adquisición de datos (TAD) y el software de procesamiento instalado en un computador. El esquema de la Fig. 1, muestra un diagrama simplificado con las partes que compone todo el sistema de adquisición del EEG. Dada la extensión del proyecto, en este artículo se describe en detalle la etapa del bioamplificador y se hace referencia al resto del sistema. 


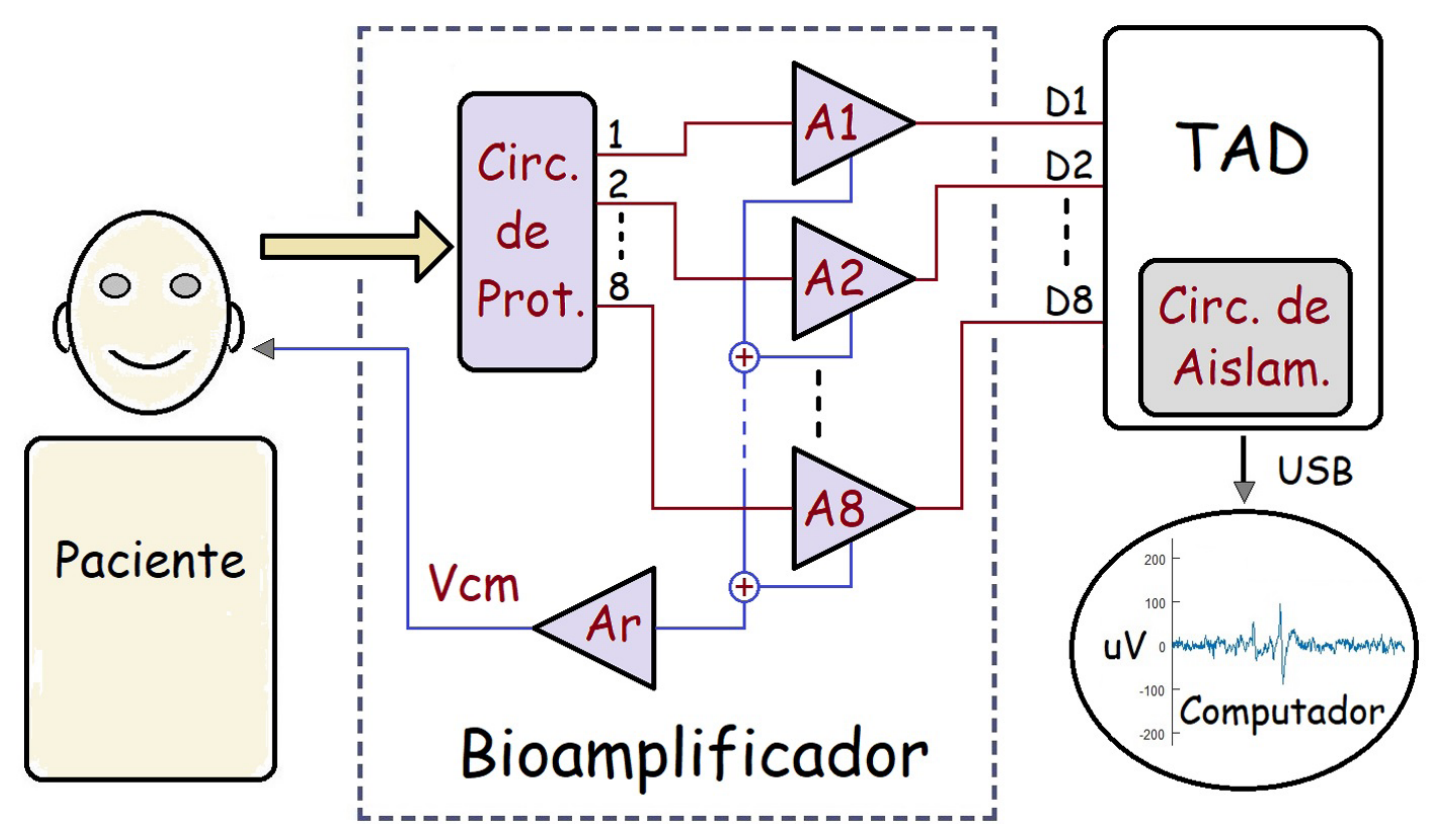

Fig. 1. Diagrama simplificado del sistema para EEG.

El producto que se presenta es un amplificador diferencial multicanal, diseñado para adquirir señales de biopotencial que se originan en el cerebro, con la finalidad de establecer las pautas que permitan el estudio de los componentes de frecuencia en un rango de 0.5 a $300 \mathrm{~Hz}$. Dado que se trata de adquirir señales de biopotencial, al hardware desarrollado se le dio el nombre de bioamplificador.

El bioamplificador se diseñó para aumentar las señales captadas de forma no invasiva, con electrodos superficiales (Flores et al., 2010). Los electrodos se colocan sobre la piel del cráneo del paciente, adoptando la configuración internacional 10-20 (Morillo, 2005). En el diagrama de la Fig. 1, se hace referencia a 8 amplificadores, desde A1 hasta A8, debido a que se manejan 8 derivaciones en forma simultánea, desde D1 hasta D8. El sistema se diseñó de 8 derivaciones por los requerimientos experimentales de este proyecto, pero la técnica aplicada puede implementarse para cualquier cantidad de canales adicionales.

La señal de cada derivación pasa por un circuito de protección de entrada y es amplificada independientemente, pero todas las señales se toman con respecto a un electrodo común, que normalmente se conecta a uno de los electrodos centrales (Fz, Cz, Pz). El amplificador Ar, permite obtener una sola señal de voltaje en modo común (Vcm), a partir de la sumatoria de las señales diferenciales de los 8 amplificadores. La señal de Vcm, normalmente se conecta en alguna región del cuerpo del paciente que sea isopotencial con respecto de los electrodos colocados en el cráneo, por lo general se conecta a un electrodo en el pabellón del oído (Navarro, 2008).

La salida del bioamplificador pasa a una tarjeta de adquisición de datos (TAD) de diseño propio (Dugarte et al., 2012), desarrollada bajo la normativa de funcionamiento y seguridad eléctrica aplicada a equipos médicos (ISO 9001, 2014). Esta TAD, permiten la digitalización multicanal de las 8 señales analógicas, D1 a D8, con velocidad de adquisición de 2000 
muestras por segundo (mps) en cada derivación y resolución de 12 bits por muestra. Los datos obtenidos se comunican al computador en tiempo real vía USB.

El procesamiento de la información se realiza en el computador bajo plataforma de software libre. El algoritmo para la adquisición de la información se desarrolla en el lenguaje de programación Pyton y el análisis de la señal se realiza implementando el software Octave. El propósito es crear una interfaz gráfica con el usuario implementando el lenguaje gráfico Gambas 3, que permita el manejo, almacenamiento y análisis de la información, bajo un formato que funcione como Historia Médica Computarizada con el estándar internacional HL7. Los antecedentes de investigación (Dugarte et al., 2015), establecen las bases experimentales que definen el protocolo a seguir.

\subsection{Descripción técnica del bioamplificador}

Cada una de las derivaciones en la etapa del bioamplificador está compuesta por circuitos electrónicos idénticos, solo difieren en el circuito de realimentación del Vcm, que es único para todas las derivaciones. El esquema de la Fig. 2, muestra el diseño del circuito eléctrico implementado en una derivación.

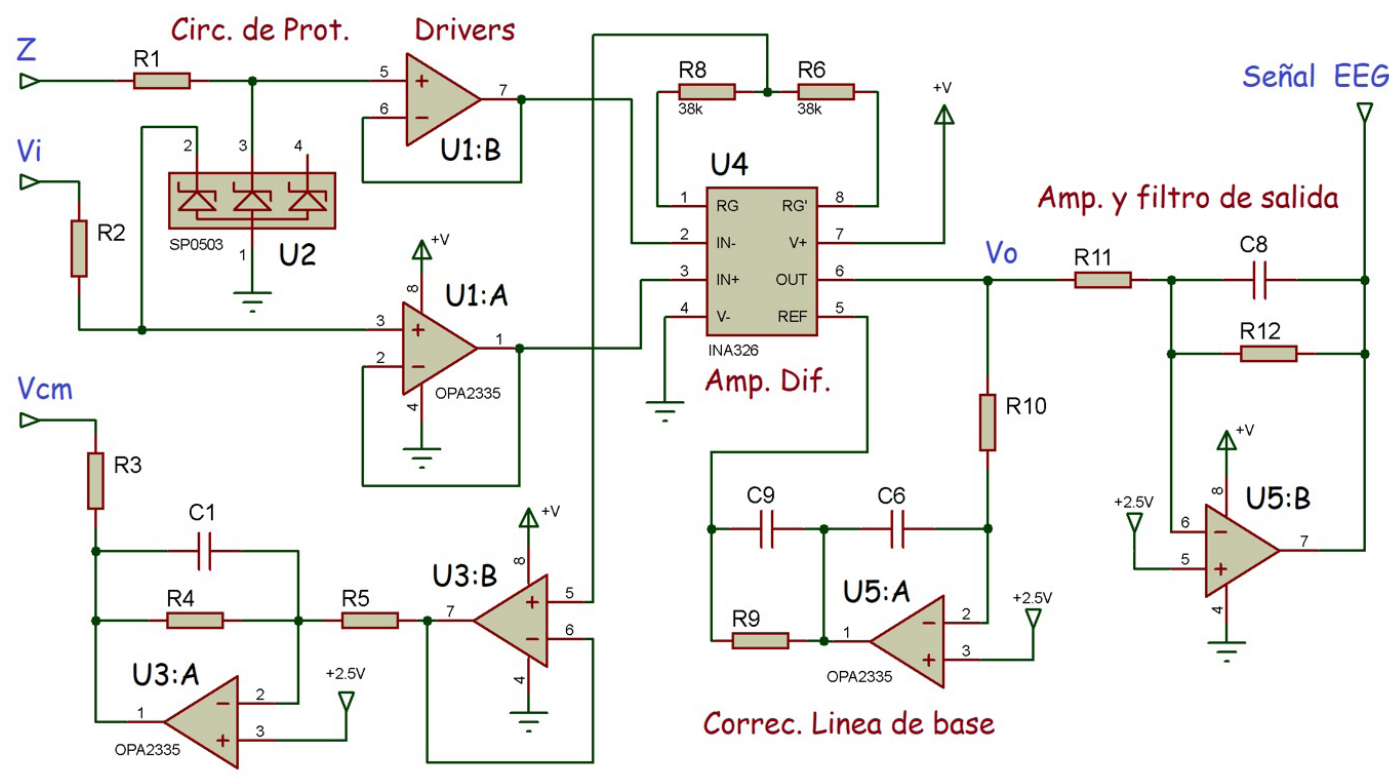

Fig. 2. Diseño del circuito eléctrico implementado en el bioamplificador.

Los diodos semiconductores encapsulados en el circuito integrado U2, son dispositivos de respuesta rápida que funcionan en contra fase. Los circuitos que forman con $\mathrm{R} 1 \mathrm{y} \mathrm{R} 2$, tienen el propósito de limitar el voltaje de entrada. Estos circuitos cumplen un doble propósito, protegen al paciente de cualquier fuga de corriente interna del aparato y protegen el instrumento contra fuentes externas de energía, como descargas de estática.

Los amplificadores operacionales U1A y U1B, conectados en configuración no inversora en cada una de las entradas, tienen la finalidad de reforzar la señal que se capta. Esto aumenta la impedancia de entrada a niveles que están por encima de $\operatorname{los} 10^{12} \mathrm{ohm}(\Omega)$, evitando que la señal de entrada se altere por el efecto de carga que pudiera ocasionar el resto del circuito. 


\subsection{Amplificación diferencial con realimentación activa}

El problema que se presenta cuando se desea adquirir alguna derivación del EEG es tratar de amplificar señales muy pequeñas, en el orden de los uV, que normalmente se encuentran contaminadas con ruidos e interferencias electrónicas (Morris, 2001). Para reducir significativamente el nivel de ruido sin afectar la información de interés, se requiere aplicar técnicas especiales en la captación y manipulación de señales. La técnica implementada en este diseño consiste en la captación de señales en modo diferencial con realimentación del voltaje modo común (Vcm por las siglas en ingles de common-mode Voltaje) (Kugelstadt, 2005), (Nash, 1998).

La señal que se desea medir está determinada por la diferencia de voltaje Vi con respecto al electrodo central Z, donde los niveles de ruido se identifican por los potenciales en modo común de Vi y Z con respecto a Vcm (ver Fig. 2). El circuito integrado U4 amplifica la señal Vi con respecto a Vcm de forma independiente a señal $\mathrm{Z}$ con respecto a Vcm. Como se identifica en la ecuación (1), el circuito integrado U4 obtiene la señal de salida Vo por la diferencia entre ambas señales. Dado que el ruido es isopotencial entre los electrodos en el cuerpo del paciente, se define que Vcm es común en ambas señales. Por consiguiente, el ruido determinado por los niveles de Vcm se atenúa y la ganancia solo magnifica la señal de interés.

$$
V o=[(V i+V c m)-(Z+V c m)] * G \rightarrow V o=(V i-Z) * G
$$

De manera ideal, la ecuación (1) señala que el Vcm se anula al realizar la diferencia entre las dos entradas, por consiguiente, el amplificador U4 solo magnifica la diferencia de potencial entre Vi y Z. En la práctica, las señales de modo común nunca serán completamente discriminadas. La especificación de la relación de rechazo de modo común "common mode rejection ratio" (CMRR), definida por la ecuación (2), es una medida del grado en el que las señales de modo común son rechazadas por el amplificador. Donde $\mathrm{Gd}$ = ganancia diferencial, $\mathrm{Gmc}$ = ganancia en modo común, y el CMRR está expresado de decibelios (dB).

$$
C M R R(d B)=20 * \log \left(\frac{G d}{G m c}\right)
$$

Los amplificadores operacionales señalados como U3A y U3B en el circuito eléctrico, tienen el objetivo de reforzar la señal de referencia Vcm que se retroalimenta al paciente (Nash, 1998). Esto se realiza con la finalidad de mejorar la relación señal - ruido y por consiguiente, aumentar la eficiencia del amplificador diferencial U4 en la eliminación del ruido que contamina la señal de biopotencial. Con este arreglo se mejora la relación de CMRR de este circuito a niveles cercanos a $100 \mathrm{~dB}$.

El valor de ganancia de U4 es 5 . El circuito se diseñó con ganancia relativamente baja debido a que su función principal es discriminar el ruido en su configuración de modo común. La ganancia total del bioamplificador se define por el producto de las ganancias de los amplificadores en cascada de todo el circuito. La ganancia requerida debe amplificar señales con amplitud máxima de $500 \mu \mathrm{V}$ pico a pico ( $\mu \mathrm{Vpp}$ ) hasta niveles óptimos para su digitalización. Dado que la característica de funcionamiento de la TAD, limita la salida del bioamplificador hasta 5 volts de amplitud pico a pico (Vpp), trae como consecuencia que, cada derivación del amplificador debe tener una ganancia de 10000. 
El circuito integrado U5:B cumple un doble propósito; sirve como amplificador en cascada con ganancia 2000 para obtener una $G$ total de 10000 y funciona como filtro pasa bajo para limitar la frecuencia máxima del ancho de banda a $300 \mathrm{~Hz}$.

\subsection{Respuesta de frecuencia del bioamplificador}

El diseño de los circuitos del bioamplificador depende de sus características dinámicas. El espectro de frecuencias de las señales de interés en la investigación, se definen dentro de un ancho de banda de 0.5 a $300 \mathrm{~Hz}$. Un filtro pasa bajo analógico (Miyara, 2004), ver Fig. 3, es un circuito eléctrico que cumple con la finalidad de atenuar el paso de las componentes alternas de la señal de entrada (Vo) que están por encima de la frecuencia de corte (fc).

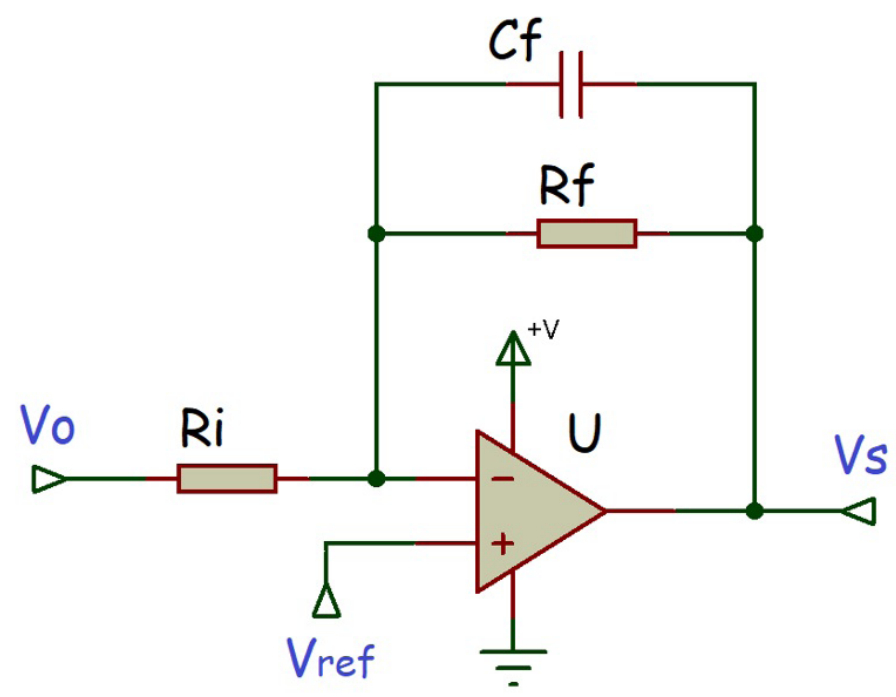

Fig. 3. Filtro pasa bajo activo de primer orden, utilizando un amplificador operacional.

La fo en un filtro paso bajo como el descrito en la Fig. 3, se define por medio de la ecuación (3). En el circuito de la Fig. 2, se puede notar que el amplificador U5:B, trabaja como filtro paso bajo, donde Cf está dado por el valor de C8 y Rf por el valor de R12.

$$
G=\frac{V S}{V o}=\frac{Z_{F}}{Z_{i}}=\frac{R f / R i}{1+j W_{0} * R f^{*} C f} \Rightarrow f_{C}=\frac{1}{2 \pi^{*} R f^{*} C f}
$$

Para efecto de cálculo en este diseño, se tomaron valores comerciales para $\mathrm{Cf}=500$ pico Faradios $(\mathrm{pF})$ y $\mathrm{Rf}=1 \mathrm{mega}$ ohm $(\mathrm{M} \Omega)$. Aplicando la ecuación (3) para calcular el filtro que utiliza el circuito U5:B, da como resultado una frecuencia de corte fc $=318 \mathrm{~Hz}$, lo cual está dentro de los requerimientos del sistema.

\subsection{Acoplamiento AC de alta eficiencia}

Asumiendo que la impedancia interna entre la referencia y la salida de U4 es nula, el circuito con el amplificador U5:A funciona de manera idéntica al descrito con U5:B. En este caso, Rf se identifica por el valor de R10, y Cf por el capacitor C6. Tomando los valores de R10 
$=1 \mathrm{M} \Omega$ y C6 $=0.33$ micro Faradio $(\mu \mathrm{F})$ se obtiene un filtro paso bajo con $\mathrm{Fc}=0.48 \mathrm{~Hz}$.

El componente DC y las frecuencias por debajo de $0.5 \mathrm{~Hz}$ no contienen información de interés en el estudio de EEG que se pretende, además representan corrimientos de la señal sobre la línea de base que resultan perjudiciales en el análisis. Para esto, la señal que deja pasar el filtro con U5:A se realimenta invertida a la referencia del amplificador de instrumentación. El efecto de U5:A en conjunto con U4 es actuar como un filtro pasa alto. Esta realimentación limita el extremo inferior del ancho de banda, atenuando las frecuencias por debajo de 0,48 Hz sin deformar la señal captada. Este circuito tiene la finalidad de ajustar continuamente el corrimiento sobre la línea de base en lo que se conoce como acoplamiento AC de alta eficiencia.

\section{Resultados y discusiones}

Debido a que los circuitos integrados presentados en el esquema de la Fig. 2, son del tipo de montaje superficial, fue necesario diseñar un circuito impreso para probar cada una de las etapas en este desarrollo. La Fig. 4, presenta el montaje físico del circuito impreso doble cara del bioamplificador desarrollado.

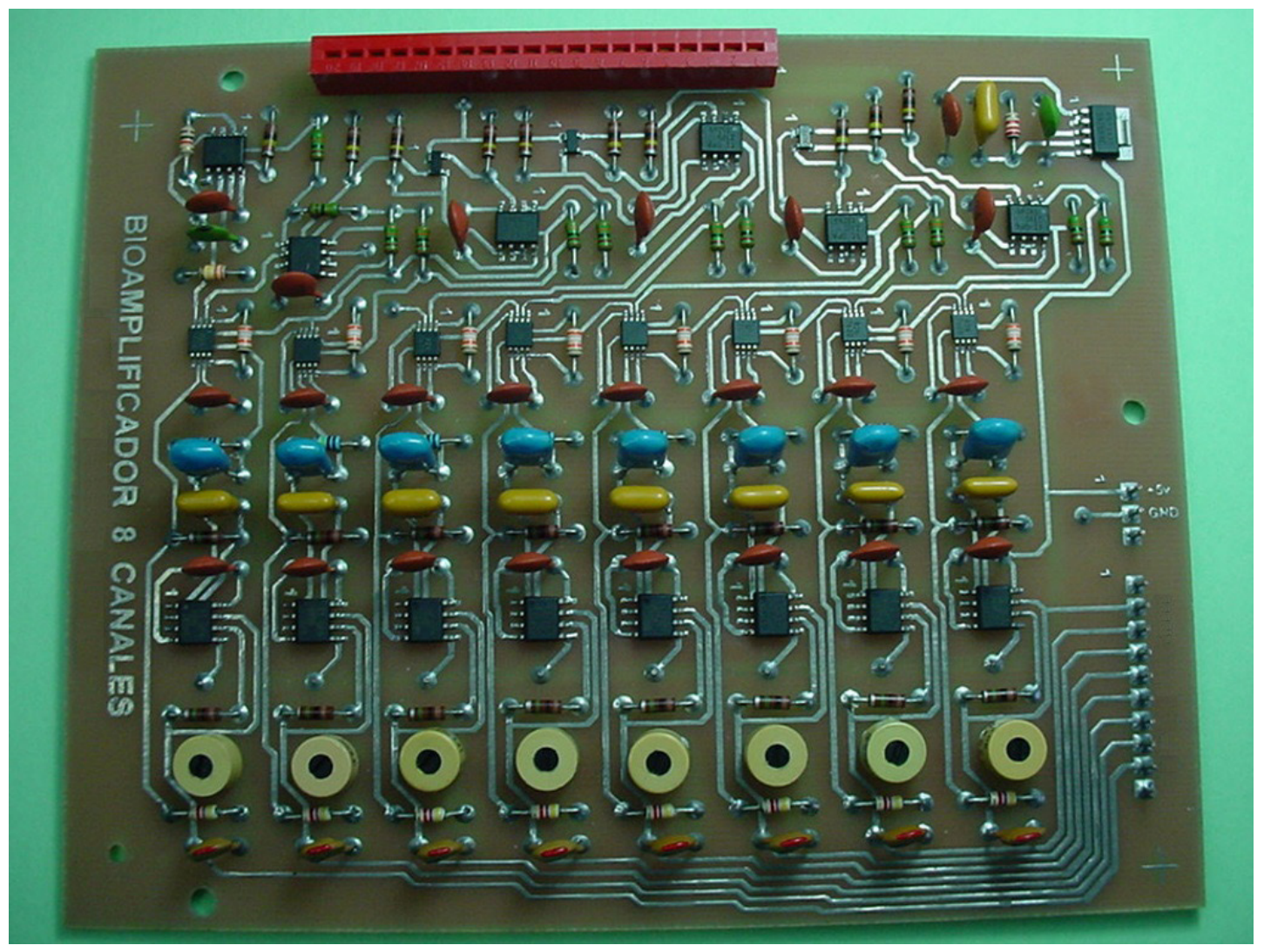

Fig. 4. Montaje físico del circuito impreso del bioamplificador

Para verificar el funcionamiento de la tarjeta desarrollada se diseñó un conjunto de pruebas que pretenden medir la respuesta de frecuencia y el nivel de error en la ganancia de amplitud en cada una de las derivaciones. Las pruebas de funcionamiento se realizaron por 
contraste, analizando una serie de adquisiciones de señales de una fuente patrón. Cada derivación del bioamplificador fue sometido a prueba de forma independiente, mientras que el resto de los canales se mantiene con las entradas en corto circuito con respecto a Vcm.

El patrón de referencia se obtuvo utilizando un atenuador con factor $G=1 / 1000$ y salida diferencial. Este atenuador fue diseñado y certificado en los laboratorios del IRB (Alvarez, 2019), con el propósito de obtener señales diferenciales con valores de amplitud en el orden de los $\mu \mathrm{V}$ a partir de un generador de señales común. La Fig. 5, muestra el esquema de conexión implementado en la evaluación de una derivación del sistema.

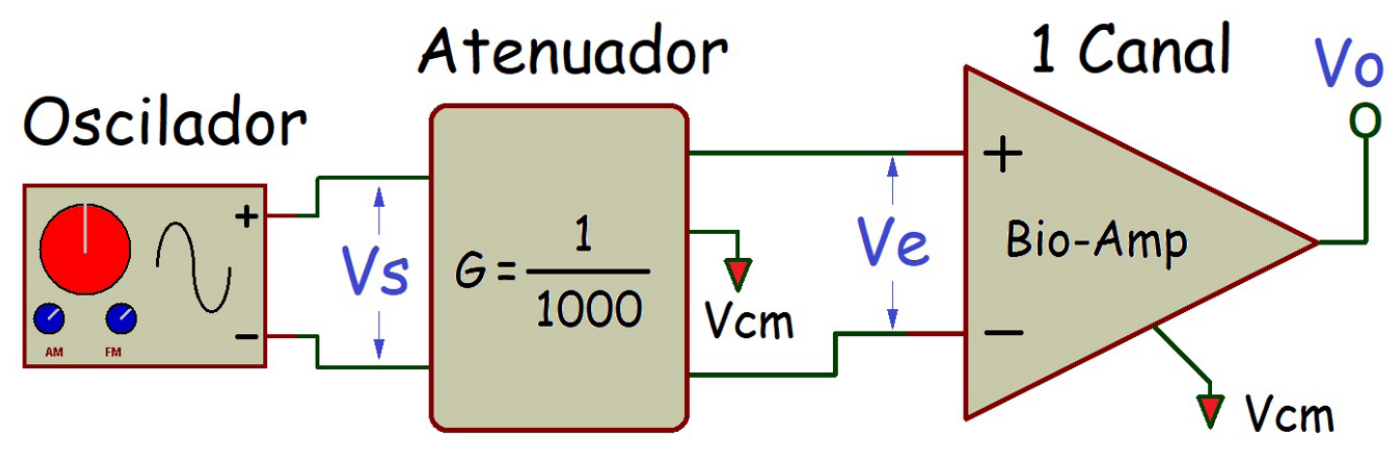

Fig. 5. Esquema de conexión implementado en la evaluación del sistema.

El generador de funciones utilizado es marca Hewlett Packard, modelo HP33120A. En las pruebas de funcionamiento se utilizó una señal sinusoidal, con amplitud constante Vs = $0.4 \mathrm{Vpp}$, lo cual permite obtener una señal diferencial $\mathrm{Ve}=400 \mu \mathrm{V}$ a la entrada del amplificador de biopotencial. La señal Ve simula los potenciales diferenciales Vi-Z con respecto a Vcm en una derivación del EEG, pero compuesto solo de señales sinusoidales y con niveles de ruido despreciables.

\subsection{Medición de error en la ganancia de amplitud}

Esta prueba tiene el propósito de contrastar los valores de amplitud de Vo en cada ciclo de la señal que pasa por el bioamplificador con el valor Ve fijado en el circuito de prueba. Se utilizó Ve con frecuencia constante de $10 \mathrm{~Hz}$ para descartar cambios de amplitud por efectos de cambio en la frecuencia, y con amplitud de $400 \mu \mathrm{Vpp}$ para contrastar el funcionamiento del amplificador al 80\% del rendimiento de la ganancia.

El procedimiento consistió en analizar 4 adquisiciones con $100 \mathrm{~s}$ de duración, realizadas mediante el empleo del sistema completo, ver Fig. 1. Cada adquisición se almacenó en el computador en un archivo con extensión "csv". Se utilizó el formato con extensión csv porque la información puede ser leída desde cualquier aplicación bajo Linux o bajo Windows, y es totalmente exportable a otros tipos de archivos como el formato "xls" (archivo de Microsoft Excel) o del tipo "txt" (archivos de texto).

El análisis de cada archivo se realizó en el computador posterior a su adquisición. Para esto, se diseñó un algoritmo programado en la herramienta de software libre denominada Octave. Este algoritmo permite medir los valores de amplitud pico a pico de cada ciclo en la señal. La Tabla 1, presenta el resultado de las mediciones obtenidas con el análisis de los 4 archivos de prueba adquiridos por el instrumento sometido a prueba. 


\begin{tabular}{|c|c|c|c|c|}
\hline & M1_sin100s.csv & M2_sin100s.csv & M3_sin100s.csv & M4_sin100s.csv \\
\hline Ciclos medidos & 1000 & 1000 & 1000 & 1000 \\
\hline $\begin{array}{c}\text { Amplitud prom. } \\
(\text { Vpp) }\end{array}$ & 3.9920 & 4.010 & 3.9990 & 4.0180 \\
\hline$\sigma$ & 0.0070 & 0.0065 & 0.0068 & 0.0064 \\
\hline $\begin{array}{c}\text { Min. Absol. } \\
(\text { Vpp) }\end{array}$ & 3.8550 & 3.8940 & 3.8720 & 3.9040 \\
\hline $\begin{array}{c}\text { Max. Absol. } \\
(\text { Vpp })\end{array}$ & 4.1350 & 4.1120 & 4.1570 & 4.1790 \\
\hline
\end{tabular}

Tabla 1. Mediciones de amplitud recopiladas en cada uno de los archivos adquiridos.

Con el sistema desarrollado, el inicio y final de adquisición se acciona manualmente. Por consiguiente, cada adquisición se realizó durante un lapso de tiempo más largo con el propósito de asegurar que se cuente con la información requerida para el análisis. El procesamiento se realizó sobre el número de ciclos señalados en la tabla para cada caso, descartando los ciclos adquiridos de más. La unidad de medición utilizada para identificar el valor medido en cada ciclo es el Vpp.

El valor de amplitud Patrón $=4.0000 \mathrm{Vpp}$, se obtiene del producto de Ve por la ganancia del amplificador. La fila identificada como "Amplitud promedio" señala la resultante de promediar el valor de amplitud del total de ciclos detectados en el archivo. La desviación estándar “ $\sigma$ ", señala la estadística de proximidad que tiene el valor promedio de amplitud al valor Patrón, con distribución normal entre todas las mediciones del archivo. Las filas encabezadas por "Min. Absol.” y "Max. Absol.”, se refieren al ciclo con menor o mayor amplitud en el registro, respectivamente.

El error absoluto (Ea) se obtiene por la diferencia entre el Patrón de referencia y el valor de amplitud en cada ciclo. El error promedio (Em) se encuentra al promediar el Ea de todas las mediciones en los 4 archivos. El error porcentual (E) se calculó aplicando la ecuación (4). La tabla 2, muestra los valores que se obtienen del análisis realizado.

$$
E=\frac{E m * 100}{\text { Patrón }}
$$

\begin{tabular}{|c|c|c|c|c|}
\hline Ciclos medidos & Ea máximo & Em & E & $\sigma$ \\
\hline 4000 & $0.1790 \mathrm{~V}$ & $0.0475 \mathrm{~V}$ & $0.1188 \%$ & 0.0067 \\
\hline
\end{tabular}

Tabla 2. Resultados obtenidos con el análisis realizado.

Los ensayos realizados en cada una de las derivaciones presentaron resultados idénticos, con variaciones numéricas en $\mathrm{E}$ inferiores al $0.03 \%$. Un error porcentual tan pequeño y con desviación estándar muy baja hacen presumir el excelente desempeño del bioamplificador en su calibración de ganancia. 


\subsection{Medición de la respuesta de frecuencia}

Esta prueba tiene el propósito de verificar experimentalmente el ancho de banda del bioamplificador. El procedimiento consistió en realizar una serie de 41 adquisiciones de señales sinusoidales, con valores de frecuencia que se incrementan progresivamente desde $0.1 \mathrm{~Hz}$ hasta $700 \mathrm{~Hz}$. Cada adquisición se realizó utilizando el esquema presentado en la Fig. 5 , con duración de 100 s y manteniendo el valor de amplitud Ve $=400 \mu \mathrm{V}$. Las mediciones de amplitud promedio en cada uno de los archivos registrados permite calcular la respuesta del sistema en $\mathrm{dB}$, por medio de la aplicación de la ecuación (5).

$$
\text { Respuesta en } d B=20 \log \left(\frac{\text { Amplitud promedio }}{\text { Patrón }}\right)
$$

La Tabla 3, muestra parte de las adquisiciones realizadas con los resultados obtenidos. Por razones de espacio no se muestra el resultado de todos los archivos adquiridos, pero los resultados presentados en la tabla evidencian la respuesta del bioamplificador a baja frecuencia.

\begin{tabular}{|c|c|c|c|}
\hline $\begin{array}{c}\text { Nombre del } \\
\text { archivo }\end{array}$ & $\begin{array}{c}\text { Frecuencia } \\
(\text { Hz) }\end{array}$ & $\begin{array}{c}\text { Amplitud Prom. } \\
(\text { Vpp })\end{array}$ & $\begin{array}{c}\text { Respuesta } \\
\text { (dB) }\end{array}$ \\
\hline M3_300mHz.csv & 0.30 & 2.17 & -5.3120 \\
\hline M4_400mHz.csv & 0.40 & 2.54 & -3.9445 \\
\hline M5_500mHz.csv & 0.50 & 2.93 & -2.7038 \\
\hline M6_700mHz.csv & 0.70 & 3.49 & -1.1847 \\
\hline M7_900mHz.csv & 0.90 & 3.72 & -0.6303 \\
\hline M8_1Hz2.csv & 1.20 & 3.90 & -0.2199 \\
\hline M9_2Hz.csv & 2.00 & 3.98 & -0.0435 \\
\hline M10_7Hz.csv & 7.00 & 4.00 & 0 \\
\hline$\ldots$ & $\ldots$ & $\ldots$ & $\ldots$ \\
\hline
\end{tabular}

Tabla 3, muestra parte de las adquisiciones realizadas con los resultados obtenidos.

La frecuencia de corte en la respuesta del sistema se identifica donde el valor de amplitud de salida supera al 70,7 \% de la señal patrón (Miyara, 2004), es decir donde Vo supera el valor de $282.8 \mu \mathrm{V}$, que equivale a $-3,012 \mathrm{~dB}$. La Fig. 6, muestra la gráfica que se genera al representar los valores de amplitud de salida en función de la frecuencia. Puede notarse que la fc calculada es muy cercana al valor de la fc real. 


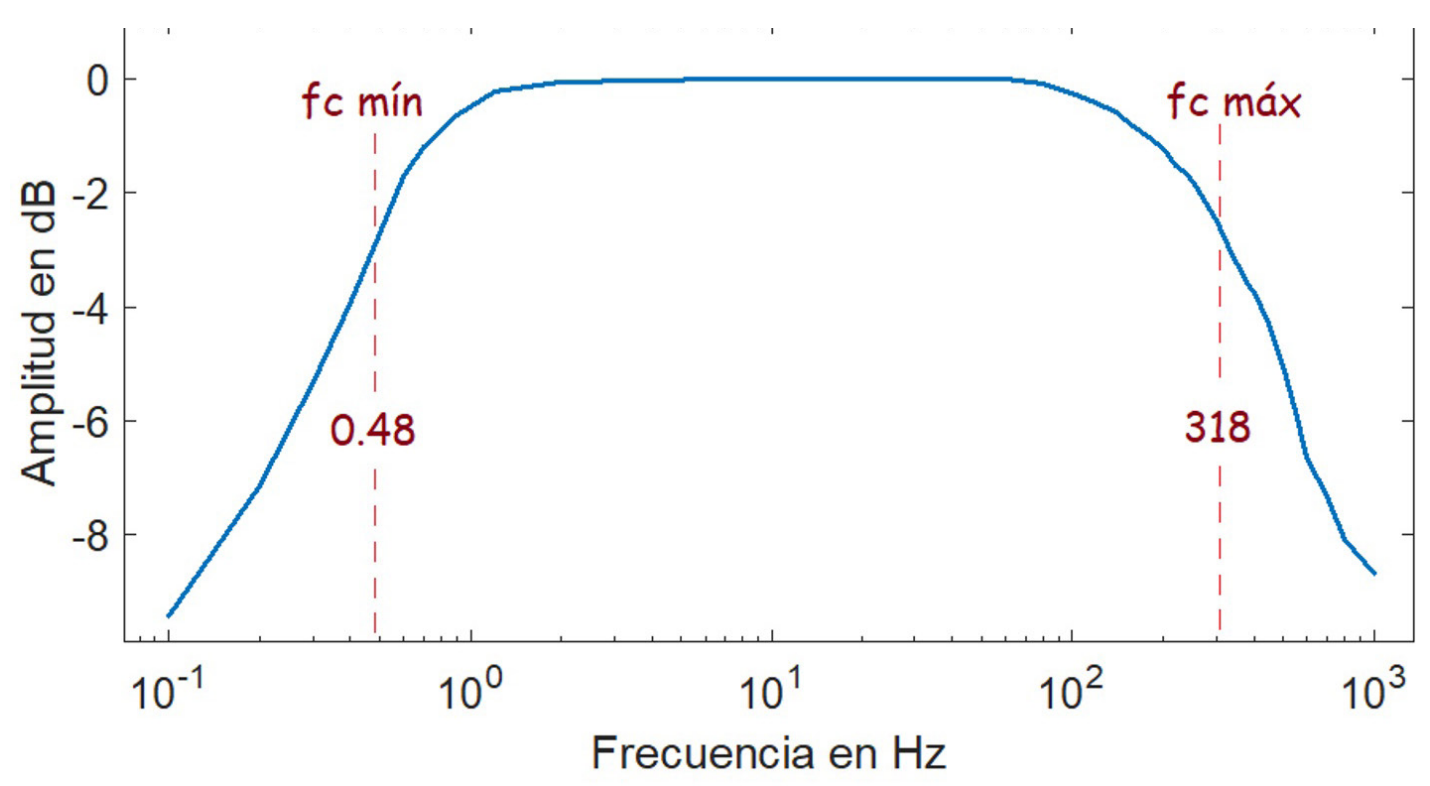

Fig. 6. Respuesta de frecuencia del bioamplificador obtenida de forma experimental.

\section{Conclusiones}

Los resultados obtenidos demuestran la eficiencia en el funcionamiento del bioamplificador desarrollado. Las señales adquiridas en los ensayos preliminares presentan una señal limpia y sin deformaciones. Los errores en la medición de amplitud presentan características idénticas en cada una de las derivaciones, con valores por debajo del $1.4 \%$. La característica de $\sigma=0,0067$ con dispersión binomial centrada demuestra la calidad en la calibración de ganancia del instrumento.

Las mediciones en la respuesta de frecuencia demostraron que, a partir de los extremos por debajo de $0,5 \mathrm{~Hz}$ y por encima de $300 \mathrm{~Hz}$, la reducción de la amplitud de salida se hace notable. Lo cual evidencia el funcionamiento de los filtros activos del circuito.

El éxito alcanzado con este desarrollo y la experiencia de certificación de la TAD realizada con anterioridad, permite predecir que es totalmente factible la implementación de este dispositivo en pacientes.

\section{Agradecimientos}

Se agradece al Instituto Regional de Bioingeniería (IRB), adjunto al Centro de Computación y Neurociencia (CeReCoN), de la Universidad Tecnológica Nacional de la República Argentina por su colaboración científica y tecnológica en el desarrollo del proyecto. También se agradece al Grupo de Ingeniería Biomédica (GIBULA) de la Universidad de Los Andes en Mérida, Venezuela por el apoyo prestado. Así mismo, se hace extensivo el agradecimiento a todas las personas e instituciones que han permitido que este proyecto sea factible. 


\section{Referencias}

Ganong W. (1999). Fisiología Médica, Manual Moderno. México, D.F.: $17^{\mathrm{a}}$ Edición, Editorial McGraw-Hill.

Tucci, A. (2004). Instrumentación biomédica. Mérida, Venezuela: departamento de publicaciones, Universidad de Los Andes.

Organización Mundial de la Salud. (2006). "Trastornos neurológicos: desafíos para la salud pública”. Disponible en http://www1.paho.org/hq/dmdocuments/2008/Trastornos_ Neurologicos.pdf.

Navarro, R. (2008). Electroencefalografía. Madrid, España. Instrumentación Biomédica Departamento Electrónica. Universidad de Alcalá.

Morillo, L. (2005). "Análisis visual del electroencefalograma”. Disponible en https://www. acnweb.org/guia/g7cap17.pdf.

Morris A. (2001). Measurement and Instrumentation Principles. Great Britain: 3ra edition, Butterworth-Heinemann Publishing Ltd.

Dugarte N., Medina R. y Rojas R. (2012). "Desarrollo del Prototipo de un Sistema de Adquisición de Datos para la Digitalización de la Señal Electrocardiográfica de Alta Resolución”. Revista del Instituto Nacional de Higiene Rafael Rangel, 43(2), 28-38.

Alvarez A., Dugarte N., Reynoso S., Dugarte E., Pérez S., Alvarado N. y Eztala D. (2019). "Desarrollo de bioamplificador de alta resolución para sistema BCI". Décimo Encuentro de Investigadores y Docentes de Ingeniería (X EnIDI), Los Reyunos, Argentina, 9 al 11 de octubre.

Alvarez, A., Alvarado, N., Reynoso, S., Gai, S., Casia, J., Pérez, S., Dugarte, E., Balacco, J., Abraham, J., Molina, A. y Dugarte, N. (2019). “Técnica de procesamiento para detección de índices que señalen la presencia de onda MU en la señal EEG”. Revista Peruana de Investigación en Salud (REPIS), 3(1), 101-108.

Dugarte, N., Álvarez, A., González, A., Pérez, S., Sánchez, J., Reynoso, S., Boverman, P., Casia, J., Martínez, D., Gracia, F., Dugarte, E., Álvarez, G. (2018). “Avances en el Proyecto para detección precoz y neurofeedback en personas con síndrome del espectro autista TEA". Quinto Congreso Internacional de Educadores en Ciencias Empíricas en Facultades de Ingeniería (ECEFI); Mendoza, Argentina, 4 y 5 de octubre.

Flores J., Becerra J. y Herrera J. (2010). "Modelo Eléctrico de un Electrodo Epimisial Implantable para la Actividad Eléctrica de un Músculo Esquelético". Journal of Vectorial Relativity, 5(4), 67-75.

ISO 9001. (2014). "Norma Internacional, ISO 13485:2003 Sistemas de gestión de la calidad Requisitos de seguridad en equipos médicos". Impreso en la Secretaría Central de ISO en Ginebra, Suiza. Número de referencia ISO 13485. 2003.

Dugarte N., Medina R., Rojas R. (2015). "Open Source Cardiology Electronic Health Record Development for DIGICARDIAC Implementation”. 11th International Symposium on Medical Information Processing and Analysis (SIPAIM), vol. 9681, Ecuador, 17 al 19 de noviembre.

Kugelstadt T. (2005). "Getting the most out of your instrumentation amplifier design". 
Analog Applications Journal, 4, 25-30.

Nash, E. (1998). A Practical Review of Common Mode and Instrumentation Amplifiers. USA: Edit Analog Devices, Copyright by Helmers Publishing Inc.

Miyara F. (2004). Filtros activos. Rosario, Argentina: Segunda Edición, Departamento de publicación de la Escuela de Ingeniería Electrónica, Universidad Nacional de Rosario.

Alvarez A., Quevedo C., Rejan S., Valls L., Perez S., Dugarte E., Dugarte N. (2019).

"Técnica de simulación de señales diferenciales de baja amplitud para certificación de bioamplificadores”. Décimo Encuentro de Investigadores y Docentes de Ingeniería (X EnIDI), Los Reyunos, Argentina, 9 al 11 de octubre. 